\title{
Peritoneal Tuberculosis Associated With Fitz-Hugh-Curtis Syndrom in a Immunocompetent Patient: Case Report
}

\author{
Fabio Freire Jose ${ }^{\mathrm{a}, \mathrm{c}}$, Gabriel Lima Lopes ${ }^{\mathrm{b}}$, Fernanda El Ghoz Leme ${ }^{\mathrm{b}}$, Ully Caroline ${ }^{\mathrm{b}}$
}

\begin{abstract}
Fitz-Hugh-Curtis syndrome is characterized by the presence of adhesions in "violin strings" between the liver and anterior abdominal wall, which is defined as perihepatitis, in association with salpingitis. The syndrome is manifested as right upper quadrant pain, possibly resulting from Glisson's liver capsule irritation. It usually disappears with an appropriate antibiotic regimen for salpingitis. So far, only gonococcal and chlamydial salpingitis have been blamed for this syndrome, as well as a report of three cases associated with genital tuberculosis. We will report a case of Fitz-Hugh-Curtis syndrome associated with peritoneal tuberculosis in an immunocompetent patient.
\end{abstract}

Keywords: Fitz-Hugh-Curtis; Tuberculosis; Peritoneum; Immunocompetent

\section{Introduction}

Disseminated tuberculosis is defined by isolation of Mycobacterium tuberculosis in blood or bone marrow and liver parenchyma or in at least two different organs [1]. It is a rare disease, especially referring to immunocompetent patients, with a higher incidence of morbidity and mortality in immunocompromised patients by various causes: HIV infection, cancer, diabetes, chronic use of immunosuppressors, chemotherapy, among others. The disseminated tuberculosis accounts for $2.8 \%$ of all tuberculosis cases and $7.3 \%$ of extrapulmonary cases [2].

\footnotetext{
Manuscript accepted for publication April 24, 2013

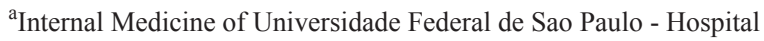
Sao Paulo, Brazil

${ }^{\mathrm{b}}$ Internal Medicine Residency Program of Hospital Sao

Paulo - Universidade Federal de Sao Paulo, Brazil

${ }^{\mathrm{c} C}$ Corresponding author: Fabio Freire Jose, Rua Napoleao de Barros,

715, Sao Paulo/SP, Brazil. Email: fabiofreire1@gmail.com
}

doi: http://dx.doi.org/10.4021/jmc1270w
Regarding peritoneal tuberculosis, it is an uncommon site for extrapulmonary infection caused by Mycobacterium tuberculosis. The risk is increased in patients with cirrhosis, HIV/AIDS, diabetes, cancer, anti-tumor necrosis factor (TNF) post-treatment and in patients undergoing continuous ambulatory peritoneal dialysis. Clinically, patients present with insidious onset of abdominal pain, ascites and fever [3].

Tuberculosis can also affect the female higher reproductive tract (uterine tubes, ovaries and endometrium) by contiguity from an intra-abdominal focus, by hematogenic read or by ascending lower genital tract (cervix, vagina and vulva). The uterine tubes and endometrium are most commonly involved. The discovery of endometrial tuberculosis always means that the tubes are infected, but tuberculous salpingitis can exist without associated endometritis. A variety of clinical manifestations have been reported, including mass or pelvic pain, infertility and abnormal uterine bleeding. In most cases, the diagnosis is made easier by endometrial biopsy to histology and culture.

Peri-hepatitis is a rare complication of disseminated tuberculosis when there is peritoneal and genital involvement. Fitz-Hugh, in 1934, characterized the clinical presentation that was described by Curtis, and he popularized it later as "Gonococcal peri-hepatitis". The eponym most commonly associated with this condition is Syndrome of Fitz-HughCurtis, a rare cause of abdominal pain, that is characterized by inflammation of the liver capsule concomitantly with pelvic inflammation without compromising the hepatic parenchyma [4]. The classic etiological agent is $N$. gonorrhoeae, however in recent years $C$. trachomatis has been detected with increasing frequency. However, there are few reports in the literature of this syndrome associated with pelvic infection by Mycobacterium tuberculosis [5, 6].

The visual diagnosis during laparoscopy may be decisive in up to $95 \%$ of cases. Normally, the visceral and parietal peritoneum are filled with multiple whitish nodules or granulomas. Other findings include enlarged lymph nodes, fibrinous strands in "violin string", and thickening of the omentum. Guided biopsies reveal caseous granulomas in up to $100 \%$ of patients and are positive for acid-fast bacilli resistant in $74 \%$ of patients $[7,8]$.

Fitz-Hugh-Curtis syndrome, as a peritoneal tuberculo- 
sis manifestation, was reported in only 3 articles previously, therefore as an atipical presentation of this disease [9]. This article's objective is to describe a case of Fitz-Hugh-Curtis syndrome as the first manifestation of disseminated tuberculosis in an immunocompetent patient.

\section{Case Report}

Female, 27 years old, came to the Emergency Room of the Hospital Sao Paulo complaining of weakness, appetite loss, weight loss of 15 kilograms, daily fever (mostly evening or night), increased abdominal size, with diffuse abdominal pain and progressive early satiety and nausea for about a month. The patient denied any prior comorbidity, medication use, previous hospitalization, recent trips and allergies. Sexually active without using condoms, reported two previous miscarriages and she was at postpartum remote.

On physical examination admission, the patient was oriented, alert, with mild psychomotor retardation, tachycardia (HR 120 bpm), tachypnea (RR 22mrpm), pale, acyanotic, anicteric and febrile (axillary temperature of $38.5^{\circ} \mathrm{C}$ ). Changes were not found in the respiratory tract justifying input signals, but she had a moderated distended abdomen, superficial reluctant to palpation in the right upper quadrant, liver palpable at $6 \mathrm{~cm}$ below the right costal margin, Traube dullness to percussion, with signs of ascites (Skoda circles, flanks dullness and Piparote signal), diffusely tender to deep palpation, with signs of peritoneal irritation (sudden decompression was present initially, but disappeared within the first days of hospitalization). Third heart sound in mitral and aortic focus was possible to be auscultated.

Upon dermatological inspection, hyperchromic lesions were identified, slightly rough, irregular, painless and nonpruritic in hypogastrium, legs and back. The gynecological examination showed the presence of bulging at the posterior fornix, and it was painful to the touch.

Laboratory tests of entry were not relevant, except for evidence of increased inflammatory activity (C-reactive protein of 295 and HCS of 82) and microcytic and hypochromic anemia with hemoglobin of 8.4 , leukocytosis of 12,100 , with normal platelet count. The Iron profile showed low levels of its soluble form $(17 \mu \mathrm{g} / \mathrm{dL})$ and high ferritin $(1,287.1 \mathrm{ng} /$ $\mathrm{mL}$ ), consistent with chronic disease anemia. Chest radiography was performed (without changes) and an abdominal ultrasound made showed an enlarged liver with regular contours and homogeneous texture and amorphous echogenic formations in adnexal regions and high volume loculated ascites. Computed Tomography of the abdomen showed hepatosplenomegaly, lymphadenopathy in diaphragmatic chains $(3.0 \times 1.4 \mathrm{~cm})$, with some favoring hypoattenuation of liquefaction, many lymph nodes in the gastrohepatic ligament and retroperitoneal vascular $(1.5 \times 1.0 \mathrm{~cm})$, diffuse thickening of the parietal peritoneum with contrast enhancement, associat- ed with moderate ascites and omental thickening, adhesions with the diaphragm and bilateral salpingitis.

A paracentesis excluded Spontaneous Bacterial Peritonitis (205 cells predominantly lymphomonocitary content, with subsequent negative culture for bacteria); cytology was negative, Lactate dehydrogenase 1,242 U/L, serum albumin gradient ascites-0.8, alchohol-acid resistant bacilli test was negative, but the Adenosine Deaminase was $70.5 \mathrm{U} / \mathrm{L}$. The patient sorological investigation for viral hepatitis, dengue, schistosomiasis, syphilis and HIV was negative, as well as the tumor markers.

The image of chest CT showed mediastinal and left mammary nodules chain, multiple small nodules center-lobular scattered in both lungs (some of them with the aspect of "tree-in-bud" appearance of inflammatory/infectious), bronchiectasis and irregular cavities in Right Lower Lobe, probably sequelae, subsegmental atelectasis in the basal regions and moderate left pleural effusion.

The patient was submitted to bronchoscopy for obtaining material for culture, since it had no respiratory symptoms and could not obtain sputum spontaneously; the examination showed no changes and bronchoalveolar search for alchohol-acid resistant bacilli and culture were negative.

Due to the strong clinical suspicion of Tuberculosis, despite polls Alchohol-acid resistant bacilli tests got negative, the antibacterial scheme was started with 4 drugs, as recommended by the Health Ministry (Rifampicin $600 \mathrm{mg}$, Isoniazid $300 \mathrm{mg}$, Pyrazinamide 1,600 $\mathrm{mg}$ and Ethambutol $1,100 \mathrm{mg})$.

Even in the first week of hospitalization, the patient developed respiratory failure with radiographic changes (suggestive of pneumonia), in addition to maintaining daily peaks of fever with negative blood cultures. Intravenous treatment with Piperacillin-Tazobactam was introduced for seven days. The patient showed significant clinical improvement on day 2 of treatment.

New serological investigation for HIV infection (including CD4 cell count and viral load) and primary immunodeficiency research were performed, but just normal results were found; autoimmunity tests were also conducted, lying just a positive ANA in title of 1: 640 nuclear fine speckled standard with unstained metaphase plate (the other anti-DNA, ANCA, anti-RNP, anti-SM, anti-Ro, anti-La and complement were normal).

We decided to move forward in the statement of probable new agent with biopsies and MRI of the abdomen, since the patient was febrile and progressively increasing the volume and abdominal pain. The MRI confirmed the earlier CT findings (hepatosplenomegaly, lymphadenomegalies, ascites and diffuse peritoneal thickening).

Liver biopsy showed only nonspecific reaction (Alchohol-acid resistant bacilli test was negative), mixed inflammatory infiltrate, predominantly lymphocytic, and hepatic steatosis grade 2. A skin biopsy revealed Cutis Macular 
Amyloidosis. A laparoscopy peritoneal and fallopian tubes biopsy was performed, but it was necessary to convert the procedure to conventional laparotomy due to technical difficulties imposed by peritoneal thickening and intense grip handles, preventing the access to the abdominal cavity.

Even now standing on the 34th day of tuberculostatic regimen, the patient had no significant improvement and she had daily fever spikes, when it was decided to start empirical treatment for Pelvic Inflammatory Disease with clindamycin and gentamicin. The gynecological examination remained without typical characteristics for Pelvic Inflammatory Disease and the vaginal smear revealed abnormal cervical type ASC-H.

The antibiotic therapy was changed again due to clinical improvement, and Doxycycline 100 mg every 12 hours, Ciprofloxacin $500 \mathrm{mg}$ every 12 hours and Metronidazole 500 mg every 8 hours were introduced (her husband was advised to take the same medications). Due to fast worsening renal function in about 45 days of hospitalization (serum creatinine of $4.9 \mathrm{mg} / \mathrm{dL}$ ), we suspended the tuberculosis medications due to the possibility of nephrotoxicity. We measured 24-hour urine proteinuria of $1.08 \mathrm{~g}$, eosinophil urine test was positive and kidneys and urinary tract ultrasound revealed increased dimensions with signs of bilateral parenchymal nephropathy. Renal biopsy showed interstices presenting diffuse lymphomono-eosinophilic inflammation and edema, non-immune pattern, which strongly suggested a diagnosis of Acute Tubulointerstitial Nephritis. However, it did not require renal replacement therapy, with progressive clinical improvement after 3 weeks. Diagnostic hypothesis of this pathology was assigned to Rifampicin or Omeprazole.

On the tenth day of the new antibiotic regimen, the patient underwent an abdomen and pelvis magnetic resonance image, which showed bilateral nephromegaly (likely by the drug nephrotoxicity), hepatosplenomegaly and aderencial process involving the uterus, ovaries and attachments, diffuse and irregular peritoneal thickening, with heterogeneous nodules in the pelvic cavity, not identifying the most massive ascites collected and displayed in previous examinations.

Finally, after three months we received the final report of peritoneal biopsies and cultures: Peritonitis with chronic fibrosing histiocytic reaction and positive for Alchohol-acid resistant bacilli. The culture of bronchoalveolar lavage fluid showed growth of Mycobacterium tuberculosis, and it was also found in liver tissue culture. The bacteria was sensitive to all tested drugs antibiogram (Rifampicin, Isoniazid, Pyrazinamide, Ethambutol and Streptomycin).

A new scheme was introduced for tuberculosis, even before results of these cultures, of an alternative way, with Streptomycin, Isoniazid, Pyrazinamide and Ethambutol. The patient was afebrile, without complaint, in good general condition, with improved appetite and weight gain. She was discharged into outpatient treatment for TB and referred to follow-up with the Gynecology and Infectious Diseases de- partments, featuring important improvement in renal function (last serum creatinine: $1.5 \mathrm{mg} / \mathrm{dL}$ ).

\section{Discussion}

First of all, we are referring to an immunocompetent young patient who presented with fever, constitutional symptoms associated with ascites and abdominal complaints. Moreover, he had risk factors for pelvic inflammatory disease, such as early onset of sexual activity, she did not use condoms, she had two miscarriages. Besides, the clinical characteristics and ascites suggested peritoneal tuberculosis, which makes the case unusual, because it is a patient with immunodeficiency research to totally negative and no other risk factors for tuberculosis were found $[10,11]$.

We noticed that in this case there was an unsatisfactory response to antituberculosis regimen employee for nearly forty days. Because of this, the envolved physicians decided to rethink the whole development of the framework so far, including all radiographs performed. From this initiative, we could observe the frame with tacks by perihepatitis with loculated ascites presented by the patient, associated with severe pelvic inflammatory activity, with adhesions and image suggestive of tubal abscess, thus creating the Fitz-HughCurtis syndrome.

Sexually transmitted agents such as Chlamydia trachomatis and Neisseria gonorrhoeae are frequently implicated in the Pelvic Inflammatory Disease, although the vaginal flora also plays an important role [12, 13]. Pelvic cellulitis and abscess after surgery, pelvic infection and pregnancyrelated pelvic infection secondary to the spread of another infection (appendicitis, diverticulitis, tumor, actinomycosis, tuberculous peritonitis, etc.) produce an image and clinical frame very similar, and in fact it's referred as Pelvic Inflammatory Disease $[14,15]$.

Thereafter, with the treatment for pelvic inflammatory disease, despite the gynecological exam that was not suggestive for Pelvic Inflammatory Disease, the goal of the medical staff was partially achieved, because the patient showed evident clinical and laboratory improvement, but it was noted that her kidney function was deteriorating.

In a study conducted in 2010 by Hong et al, with the primary diagnosis of peri-hepatitis in association with tuberculosis of 101 cases, the authors diagnosed the condition by laparoscopy, and only two of them were related to pelvic tuberculosis [4]. The first literature description of Fitz-HugCurtis syndrome associated with tuberculosis was made in 2003 by Sharma et al, who reported three cases [5]. In 2007, also Sharma et al, described 40 cases of this syndrome in laparoscopic investigation of 82 patients with genital tuberculosis, thus demonstrating a high prevalence of peri-hepatitis among patients with genital tuberculosis (48.7\%) [6].

Genital tuberculosis diagnosis is often difficult, and a 
high clinical suspicion must exist for an approach that enables the diagnosis invasive workup [16, 17]. Laparoscopy can be very useful in the etiological investigation of tuberculous peritonitis, since the research of Alchohol-acid resistant bacilli in ascites is often negative, a positive culture ranges from 20 to $80 \%$ and it can take months to get the result, and PCR has a sensitivity variable and may not be available in all centers [18]. In this case report, the patient had a clinically suggestive case, but the laparoscopic biopsy was essential for diagnosis, noting that the clinical suspicion is mandatory for the treatment, even without microbiological evidence that we obtained during the investigation.

\section{Conclusion}

Peritoneal tuberculosis should be considered in all patients with lymphocytic ascites, with a gradient of serum-ascites albumin $<1.1 \mathrm{~g} / \mathrm{dL}$. Diagnosis can be difficult since the disease is insidious and it can have a variable presentation.

Although even with the diagnosis of tuberculous peritonitis, the patient did not initially responded to the antituberculosis treatment and she dramatically improved with treatment for Pelvic Inflammatory Disease. About 90 days later, peritoneal biopsy initially had no caseous granulomas showed positive for -acid resistant bacilli test; cultures for M. tuberculosis in liver sample and bronchoalveolar lavage were also positive. This paper reports, therefore, multi-bacterial association of peritoneal tuberculosis and Fitz-HughCurtis syndrome in an immunocompetent patient, in a context of disseminated mycobacteriosis.

\section{References}

1. Iseman MD. Extrapulmonary tuberculosis in adults. In: A clinician's guide to tuberculosis. 1st ed. Philadelphia: Lippincott Williams \& Wilkins, 2000:145-197.

2. Charfi MR, Dougui MH, Louzir B, Mestiri A, Zbiba M, Belalgia MS, Daghfous J. [Disseminated tuberculosis in non-immunocompromised host: three case reports]. Rev Med Interne. 1998;19(12):917-920.

3. Golden MP, Vikram HR. Extrapulmonary tuberculosis: an overview. Am Fam Physician. 2005;72(9):17611768.

4. Hong DG, Choi MH, Chong GO, Yi JH, Seong WJ, Lee YS, Park IS, et al. Fitz-Hugh-Curtis Syndrome: single centre experiences. J Obstet Gynaecol. 2010;30(3):277280.

5. Sharma JB, Malhotra M, Arora R. Fitz-Hugh-Curtis syn- drome as a result of genital tuberculosis: a report of three cases. Acta Obstet Gynecol Scand. 2003;82(3):295-297.

6. Sharma JB, Roy KK, Gupta N, Jain SK, Malhotra N, Mittal S. High prevalence of Fitz-Hugh-Curtis Syndrome in genital tuberculosis. Int J Gynaecol Obstet. 2007;99(1):62-63.

7. Martins HS, Neto RAB, Neto AS, Velasco IT. Emergencias Clinicas. Abordagem pratica. 7a ed. rev. e atual. Barueri, SP: Manole, 2012.

8. Demir K, Okten A, Kaymakoglu S, Dincer D, Besisik F, Cevikbas U, Ozdil S, et al. Tuberculous peritonitis-reports of 26 cases, detailing diagnostic and therapeutic problems. Eur J Gastroenterol Hepatol. 2001;13(5):581585.

9. Tanrikulu AC, Aldemir M, Gurkan F, Suner A, Dagli CE, Ece A. Clinical review of tuberculous peritonitis in 39 patients in Diyarbakir, Turkey. J Gastroenterol Hepatol. 2005;20(6):906-909.

10. Curtis AH. A cause of adhesions in the right upper quadrant. JAMA. 1930 94(16):1221-1222.

11. Pels RJ, Bor DH, Woolhandler S, Himmelstein DU, Lawrence RS. Dipstick urinalysis screening of asymptomatic adults for urinary tract disorders. II. Bacteriuria. JAMA. 1989;262(9):1221-1224.

12. Aliyu MH, Aliyu SH, Salihu HM. Female genital tuberculosis: a global review. Int J Fertil Womens Med. 2004;49(3):123-136.

13. Tinelli A, Malvasi A, Vergara D, Martignago R, Nicolardi G, Tinelli R, Pellegrino M. Abdominopelvic tuberculosis in gynaecology: laparoscopical and new laboratory findings. Aust N Z J Obstet Gynaecol. 2008;48(1):9095.

14. Haight JB, Ockner SA. Chlamydia trachomatis perihepatitis with ascites. Am J Gastroenterol. 1988;83(3):323325.

15. Fitz-Hugh T Jr. Acute Gonococcic Peritonitis of right upper quadrant in women. JAMA. 1934 102:2094-2096.

16. Sanai FM, Bzeizi KI. Systematic review: tuberculous peritonitis--presenting features, diagnostic strategies and treatment. Aliment Pharmacol Ther. 2005;22(8):685700.

17. Poyrazoglu OK, Timurkaan M, Yalniz M, Ataseven H, Dogukan M, Bahcecioglu IH. Clinical review of 23 patients with tuberculous peritonitis: presenting features and diagnosis. J Dig Dis. 2008;9(3):170-174.

18. Peipert JF, Boardman LA, Sung CJ. Performance of clinical and laparoscopic criteria for the diagnosis of upper genital tract infection. Infect Dis Obstet Gynecol. 1997;5(4):291-296. 